\title{
Nomograms for incident risk of postpartum type 2 diabetes in Chinese women with prior gestational diabetes mellitus
}

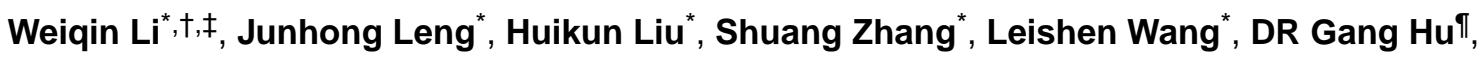 \\ and DR Jie Mi†, \\ *Tianjin Women's and Children's Health Center, Tianjin, China \\ † Department of Epidemiology, Capital Institute of Pediatrics, Beijing, China \\ ‡ Graduate School of Peking Union Medical College, Beijing, China \\ I Pennington Biomedical Research Center, Baton Rouge, Louisiana, USA
}

\section{Summary}

Objective-Counseling patients with gestational diabetes mellitus (GDM) on their individual risk of postpartum type 2 diabetes (T2D) is challenging. This study aimed to develop nomograms for predicting incident risk of postpartum T2D in women with GDM diagnosed by WHO1998 criteria.

\begin{abstract}
Methods-We performed a retrospective cohort study in 1,263 Chinese women with GDM, of whom 83 were diagnosed as T2D at 2.3 years postpartum. Multivariate Cox proportional hazards models were used to investigate the independent predictors for postpartum T2D. The results of multivariate analyses were used to formulate nomograms for predicting incident risk of postpartum T2D. The predictive accuracy was evaluated using the area under the receiver operating characteristic curve (AUROC).
\end{abstract}

Results-On multivariate analysis, independent predictors of postpartum T2DM in women with GDM included family history of diabetes [hazard ratio (HR) and its 95\% confidential interval (95\% CI): 2.06 (95\% CI: 1.32-3.22)], history of pregnancy-induced hypertension [3.11 (95\% CI: 1.86-5.21)], pre-pregnancy BMI [1.00, 1.90 (95\%CI: 1.14-3.16), and 3.67 (95\%CI: 2.03-6.63) for BMI $<24,24-28$, and $\left.\geq 28 \mathrm{~kg} / \mathrm{m}^{2}\right]$, and 2-hour glucose at 26-30 gestational weeks [1.00, 2.84 (95\%CI: 1.42-5.69), and 9.42 (95\%CI: 4.46-19.90) for 2 hour glucose at $7.8 \sim<8.5,8.5 \sim<11.1$, and $\geq 11.1 \mathrm{mmol} / \mathrm{L}$ ). The overall AUROC of nomogram was $82.8 \%$ (95\%CI: $78.1 \%-87.5 \%)$, with AUROCs of $85.9 \%$ (95\%CI: $79.7 \%-92.1 \%$ ) and $83.2 \%$ (95\%CI: $77.9 \%-88.6 \%$ ) for postpartum 2year and 3-year risk of T2D, respectively.

Corresponding author at: Chronic Disease Epidemiology Laboratory, Pennington Biomedical Research Center, Baton Rouge, LA 70808, USA, gang.hu@ pbrc.edu (G. Hu) or Department of Epidemiology, Capital Institute of Pediatrics, Beijing 100020, China, jiemi@vip.163.com (J. Mi).

Authors' Contributions

Weiqin Li designed the study, acquired the data, performed the statistical analysis, interpreted the data, drafted the article, and approved the final version to be published. Jie Mi and Gang Hu designed the study, reviewed and critically revised the article, and approved the final version to be published. Huikun Liu, Shuang Zhang, Leishen Wang, Junhong Leng, and Gang Hu performed the field work and critically revised the article and approved the final version to be published. Gang Hu and Jie Mi was responsible for the integrity of the work as a whole.

Competing Interests

All authors declare that they have no conflict of interests relevant to this article to disclose. 
Conclusions-This easy-to-use nomogram, with noninvasive clinical characteristics, can accurately predict the risk of postpartum T2D in women with GDM. It may facilitate risk communication between patients and clinicians.

\section{Keywords}

nomograms; postpartum Type 2 Diabetes; gestational diabetes mellitus

\section{Introduction}

The global prevalence of type 2 diabetes (T2D) has increased significantly in recent decades.

${ }^{1}$ The latest China National Survey has showed that the prevalence of diabetes (a selfreported previous diagnosis by health care professionals, fasting plasma glucose levels $\geq 7$ $\mathrm{mmol} / \mathrm{l}$, 2-hour plasma glucose levels $\geq 11.1 \mathrm{mmol} / \mathrm{l}$, or $\mathrm{HbA} 1 \mathrm{c}$ concentration $26.5 \%$ ) and prediabetes (fasting plasma glucose levels between $5.6 \mathrm{mmol} / \mathrm{l}$ and $7.0 \mathrm{mmol} / \mathrm{l}$, 2-hour plasma glucose levels between $7.8 \mathrm{mmol} / \mathrm{l}$ and $11.1 \mathrm{mmol} / \mathrm{l}$, or HbA1c concentrations between $5.7 \%$ and $6.4 \%$ in participants without a prior diabetes diagnosis) according to the American Diabetes Association (ADA) 2010 criteria were $11.6 \%$ and $50.1 \%$ among adults, respectively. ${ }^{2}$ The growing T2D burden will inevitably present an overwhelming challenge in terms of health service resources around the world, including China. Early identification of person at a high risk of T2D is a crucial issue to be resolved.

Gestational diabetes mellitus (GDM), defined as any degree of glucose intolerance with onset of or first recognization during pregnancy, ${ }^{3}$ has been recognized as an independent risk factor of postpartum T2D. In 2009, a meta-analysis showed that women with GDM diagnosed by different criteria had a 7.43-fold risk of T2D compared with those who had a normoglycemic pregnancy. ${ }^{4}$ As a high-risk population, women with GDM should draw more attention to prevent the occurrence of postpartum T2D.

Risk prediction models have considerable potential to the decision-making process regarding the management of T2D patients. More than 40 risk prediction models for T2D have been developed among different populations according to Collins's review. ${ }^{5}$ However, despite the large number of risk prediction models, only a very small minority were routinely used in clinical practice. ${ }^{5}$ In addition to the heterogeneity between different populations and complex mathematical formulas, ${ }^{6-11}$ lack of simple and visual tools to promote the usage of these prediction risk models would be another important barrier in risk communication between patients and clinicians. Moreover, most of risk prediction models for T2D were conducted among general population. Up to now, no tools have been developed to predict postpartum T2D among women with GDM. Therefore, the present study aimed to develop simple, fast, and noninvasive tools to identify postpartum T2D risk among women with GDM. 


\section{Materials and methods}

\section{Study population}

A retrospective cohort study was performed in 1,263 GDM women one to five years postpartum using the data of the participants from the Tianjin Gestational Diabetes Mellitus Prevention Program. ${ }^{12-16}$ The details of the program have been described previously. ${ }^{13}$ Since 1999, all pregnant women living in the 6 central urban districts in Tianjin have participated in universal screening for GDM, and the average proportion of screened pregnancies was $>91 \%$ from 1999 to $2008 .{ }^{17}$

All pregnant women at 26-30 weeks' gestation participated in a 1-h 50-g glucose screening test (GST), and those who had a glucose reading $\geq 7.8 \mathrm{mmol} / \mathrm{L}$ were invited to undergo a 75 g glucose 2-h oral glucose tolerance test (OGTT) at Tianjin Women's and Children's Health Center. ${ }^{17}$ GDM was defined using the World Health Organization (WHO) 1998 criteria. ${ }^{18}$ Women with a 75-g glucose 2-h OGTT result confirming either diabetes (fasting glucose $\geq 7$ $\mathrm{mmol} / \mathrm{l}$ or 2-h glucose $\geq 11.1 \mathrm{mmol} / \mathrm{l}$ ) or impaired glucose tolerance (2-h glucose $\geq 7.8$ and $<11.1 \mathrm{mmol} / \mathrm{l}$ ) are regarded as having GDM.

All pregnant women who were diagnosed with GDM between 2005 and $2009(\mathrm{~N}=4,644)$ were recruited 1-5 years after delivery, based on a health care registration system providing health and contact information for mothers with GDM ${ }^{13}$. Finally, 1,263 women with GDM returned. Off them, 114 had diabetes and 1149 were IGT by the WHO 1998 GDM criteria based on the OGTT done in 26-30 gestational week. Further results to confirm if these women had returned to normoglycaemia at the end of pregnancy were not available to use. There were no differences between the women with GDM on the 26-30 gestational week OGTT who returned and those who did not return when comparing age (28.9 vs. 28.7 years), fasting glucose ( 5.34 vs. $5.34 \mathrm{mmol} / \mathrm{L})$, 2-h glucose ( 9.23 vs. $9.16 \mathrm{mmol} / \mathrm{L})$, and the prevalence of impaired glucose tolerance $(90.9 \%$ vs. $91.8 \%)$ and diabetes $(9.1 \%$ vs. $8.2 \%) .{ }^{14}$

The study was approved by the Human Subjects Committee of the Tianjin Women's and Children's Health Center; and informed consent was obtained from each participant.

\section{Data collection}

After informed consent, all women received a face-to-face interview by trained physicians with structured questionnaire and a series of physical examination. The questionnaire included basic information (including race, marriage, education, income, occupation), family history of T2D, self-reported pre-pregnancy weight, pregnancy information (age at pregnancy, and pregnancy-induced hypertension), and postpartum information (years after delivery, etc). Values of fasting and 2-h glucose during a 75-g glucose OGTT at 26-30 gestational weeks were copied from the Tianjin Women's and Children's Health Center's Central Lab' records. The physical examination included blood pressure measured in the sitting position, height, weight, and waist circumference determined at the umbilicus with the subject standing. Body mass index (BMI) was calculated by dividing the weight in kilograms by the height in meters squared. All women further received examinations of an overnight fast glucose and a 2-hour OGTT. According to American Diabetes Association 
(ADA)'s criteria, ${ }^{19}$ postpartum T2D was defined as fasting glucose $\$ .0 \mathrm{mmol} / \mathrm{l}$ or 2 -h glucose $\forall 1.1 \mathrm{mmol} / \mathrm{l}$.

\section{Statistics methods}

Two steps were conducted to determine the predictors of postpartum T2D risk. First, chisquare tests and variance analyses were used to test the crude associations between each selected risk factor (family history of diabetes, pre-pregnancy body mass index [BMI], age at pregnancy, pregnancy-induced hypertension, and 2-hour glucose during a 75-g glucose OGTT at 26-30 gestational weeks) and the risk of postpartum T2D. Second, multivariate Cox proportional hazards regression models including all independent index variables were conducted to determine the final predictors of postpartum T2D models with backward variable-elimination strategy. Hazard ratios (HRs) and 95\% confidential intervals (95\% CIs) were calculated to estimate relative risk of postpartum T2D for each predictor.

In order to develop an easy-to-use risk prediction tool, regression coefficients of postpartum T2D were rounded into integer risk scores, ${ }^{20}$ and predicted risk for each risk score was calculated and depicted in nomograms according to the method developed by Iasonos et al. ${ }^{21}$ In addition to the overall AUROC for the overall predictive accuracy of nomogram, the predictive accuracies of nomograms were further assessed with AUROCs for postpartum 2year and 3-year risk of T2D. Moreover, the calibration accuracies of nomograms were assessed by plotting of predicted probabilities from the nomograms versus the actual probabilities of T2D at postpartum 2-year and 3-year, respectively.

All statistical analyses were performed using R software (version 3.4.2). Cox regression, nomogram development, and AUROC evaluations were conducted with packages of 'survival' (version 2.41-3), 'rms' (version 5.1-1), and 'pROC' (version 1.10.0), respectively. A two-tailed $\mathrm{P}$ value of less than 0.05 was considered to indicate statistical significance.

\section{Results}

\section{General characteristics}

During a median follow-up period of 2.3 years after delivery, 83 T2D cases were identified. Compared with women without T2D, women with diagnosed T2D at time of follow up were more likely to have family history of diabetes and history of pregnancy-induced hypertension, had significantly higher values of pre-pregnancy BMI, and 2-hour glucose during an OGTT at 26-30 gestational weeks (Table 1).

\section{Predictors of postpartum T2D among women with GDM}

As shown in table 2, several factors before or during pregnancy, including family history of diabetes (HR: 2.06, 95\% CI: 1.32-3.22), history of pregnancy-induced hypertension (HR: 3.11, 95\%CI: 1.86-5.21), pre-pregnancy BMI [HRs for BMI <24, 24-28, and $28 \mathrm{~kg} / \mathrm{m}^{2}$ : 1.00, 1.90 (95\% CI: 1.14-3.16), and 3.67 (95\%CI: 2.03-6.63), respectively], and 2-hour glucose at 26-30 gestational weeks [HRs for 2 hour glucose $7.8-<8.5,8.5-<11.1$, and $\geq 11.1$ $\mathrm{mmol} / \mathrm{L}: 1.00,2.84$ (95\%CI: 1.42-5.69), and 9.42 (95\%CI: 4.46-19.90), respectively] were positively and significantly associated with the risk of incident postpartum T2D in the cox 
proportional hazards model. The HRs for postpartum 2-year and 3-year risk of T2D associated with potential risk factors were similar with overall results, especially for postpartum 3-year risk of T2D (Table 2).

\section{Predictive accuracy and calibration accuracy of nomogram for postpartum T2D risk}

Based on results of multivariable analyses, nomogram for postpartum risk of T2D in women with GDM were formulated in figure 1. The overall predictive accuracy of this nomogram was $82.8 \%$ (95\%CI: $78.1 \%-87.5 \%)$. Moreover, the predictive accuracies of nomograms for postpartum 2-year and 3-year risk of T2D were 85.9\% (95\%CI: 79.7\%-92.1\%) and 83.2\% (95\% CI: 77.9\%-88.6\%), respectively. As shown in Figures 2A and 2B, the observed and predicted probabilities for both postpartum 2-year and 3-year risk of T2D fell along the 45degree line, which suggested relatively well calibration accuracies of this nomogram for both postpartum 2-year and 3-year risk of T2D.

\section{Discussion}

This is the first study to develop postpartum T2D risk scores including several factors before/during pregnancy among women with GDM. Our study showed that the discriminations of postpartum T2D among women with GDM were very good, and very similar to the previously reported risk prediction models. ${ }^{6-8,22}$

More than 40 risk prediction models for T2D have been developed among different populations. ${ }^{5}$ However, only a very small minority were routinely used in clinical practice, ${ }^{5}$ due to the heterogeneity between different populations, complex mathematical formulas, 6-11,22 and lack of simple and visual tools to promote the usage of these prediction risk models. Our study is the first to visualize these prediction models with nomograms. Nomograms are a pictorial representation of a complex mathematical formula, ${ }^{23}$ and are widely used as a frequently-used tool in oncology prognosis. ${ }^{23-26}$ However, few studies have focused on the development of this easy-to-use tool for T2D risk prediction. With the ability to generate an individual probability of T2D by integrating diverse risk predictors, nomograms meet our desire for visual tools and fulfill our drive towards personalized prevention. Through user-friendly digital interfaces, increased accuracy, and more easily understood risk-prediction compared with conventional mathematical formula, rapid computation nomograms allow for seamless incorporation of risk assessment to clinical decision making. ${ }^{26}$ With these simple, fast, inexpensive, and noninvasive tools, we would expect to effectively identify individuals who are at an increased risk of postpartum T2D after GDM. Then, health care interventions, lifestyle changes, diagnosis management and treatment can be initiated and ultimately improve patient outcomes.

In addition to the user-friendly graphical interfaces for facilitating the clinical decision making, nomograms also directly reflect the relative importance of predictors in models. As shown in figure 1, 2-hour 75-g glucose level at 26-30 gestational weeks was the well-known strongest predictors for postpartum T2D after GDM. Similar with previous prediction models reported among general population, ${ }^{5}$ pre-pregnancy BMI, family history of diabetes and history of pregnancy-induced hypertension were also significantly associated with postpartum T2D among women with GDM. For pre-pregnancy BMI, previous studies 
showed that Asians and Asian Americans are more likely to develop GDM and diabetes compared with Americans and Europeans, though they generally have lower BMI levels than whites. ${ }^{27,28}$ Two reasons had been put forward to explain this phenomenon. Compared with their counterparts, Asians not only have higher adiposity per unit BMI, ${ }^{29}$ but also have decreased insulin sensitivity and increased $\beta$-cell dysfunction compared with whites. ${ }^{30,31}$ Our previous studies also supported the association of increased risk of postpartum diabetes and prediabetes with pre-pregnancy obesity, ${ }^{12}$ and also found the potential different etiologies of diabetes risk between obese and non-obese Asians women after GDM. ${ }^{14}$ In the future, more studies are needed to validate our results found among women with GDM.

This study aimed to develop a model to predict the future incident risk of postpartum T2D in women with GDM based on a cohort study. Thus, the potential risk predictors for incident T2D after delivery included variables before or during pregnancy, but did not include postpartum variables such as postpartum weight changes and breast feeding, etc. As the next step, our team will develop a further PREDICTION model to indicate the risk of prevalent T2D based on a cross-sectional study, and potential risk factors could then include both variables before/during pregnancy and variables after delivery.

Women with GDM have a significantly increased risk of developing type 2 diabetes after delivery. In 2002, Kim et al did a meta-analysis including 28 studies with the follow-up period ranged from 6 weeks to 28 years postpartum from 1965 to 2001, and the risk of developing T2D was $2.6 \%$ to $70.0 \%{ }^{32}$ The wide variation in cumulative diabetes incidence resulted from the differences of follow-up length and frequency, GDM diagnostic criteria, population, and whether preexisting diabetes first detected during their pregnancy was counted as GDM. In 2009, Bellamy et al meta-analyzed 20 studies from 1960 to 2009, and the study with the largest number of GDM reported the incidence of T2DM as 3.7\% at 9 months postpartum and $18.9 \%$ at 9 years postpartum, respectively. ${ }^{4}$ In our study, the rate of T2D was $6.6 \%$ at median 2.3 years postpartum. ${ }^{13}$ There were two reasons for the high incidence of T2D. First, GDM was defined using the 1998 WHO's criteria, and including both diabetes (fasting glucose $\geq 7 \mathrm{mmol} / \mathrm{L}$ or 2 -h glucose $\geq 11.1 \mathrm{mmol} / \mathrm{L}$ ) and IGT (2-h glucose $\geq 7.8$ and $<11.1 \mathrm{mmol} / \mathrm{L}$ ) during pregnancy. The 1263 pregnant women included 114 diabetes and 1149 IGT women during pregnancy, and the incident rate of postpartum T2D was $26.3 \%$ and $4.6 \%$, respectively. The preexisting diabetes first detected during pregnancy would significantly increase the risk of postpartum T2D. Results showed that the multiple adjusted risk of postpartum T2D among women with 2 hour glucose at 26-30 gestational weeks $\geq 11.1 \mathrm{mmol} / \mathrm{L}$ was 9.42 -fold of those with 2 hour glucose at $26-30$ gestational weeks between 7.8 and $8.5 \mathrm{mmol} / \mathrm{L}$. The second reason was that most of GDM women did not take special measures, such as monitoring blood glucose, controlling body weight, or improving lifestyle, to reduce the risk of postpartum T2D after delivery before 2010 .

The nomogram of this study is conducted among Chinese women with GDM. Similar with the various prediction models for type 2 diabetes, the ethnicity and the heterogeneity of study population will affect the extending of our prediction model. Moreover, the cumulative incidence of diabetes in women with a previous GDM is also affected by the duration of follow-up. Besides the overall postpartum risk of T2D, we also developed the prediction models for postpartum 2-year and 3-year risk of T2D. The definition of GDM during 2005- 
2009 in the present study used the WHO 1998 criteria. ${ }^{18}$ Since 2010, the International Association of Diabetes and Pregnancy Study Groups (IADPSG) criteria has been recommended to supercede the WHO's criteria. ${ }^{33}$ The lower threshold of IADPSG for fasting glucose ( $\geq 5.1 \mathrm{mmol} / \mathrm{L}$ ) and adding 1 hour glucose have resulted in more cases of GDM than in the past. However, we did not have one hour glucose measurements and so we were unable to reanalyze our data and prediction based on the IADPSG criteria.

There were other limitations. First, as other risk prediction tools, lack of objective and a unbiased external validation on a different data set is also a common concern in this study. Second, although our analyses have included an extensive set of potential predict factors, there were still other unmeasured factors such as insulin secretion. Third, some information including pre-pregnancy BMI and pregnancy information including history of pregnancyinduced hypertension were obtained using questionnaire, which might produce recall bias and lead to underestimations. Despite these limitations, this study is still the first large population-based cohort study of predicting risk of T2D after GDM.

\title{
Conclusions
}

In conclusion, women with GDM are facing an increasing risk of T2D after delivery. Nomogram for postpartum T2D after GDM was very effective and easy-to-use tools to identify individuals who are at an increased risk of T2D after GDM. Based on this tool, personalized health care interventions and lifestyle changes should be initiated and targeted at those high-risk population and ultimately improve their outcomes.

\section{Acknowledgments}

\author{
Funding
}

This project was supported by the grants from the National Key Research and Development Program of China (Grants No: 2018YFC1313903, 2016YFC1300101 and 2016YFC0900602), National Natural Science Foundation of China (Grant No: 81502821), the European Foundation for the Study of Diabetes (EFSD)/Chinese Diabetes Society (CDS)/Lilly Program for Collaborative Research between China and Europe, Tianjin Women's and Children's Health Center, and Tianjin Public Health Bureau. Dr. Gang Hu was partly supported by the grant from the National Institute of Diabetes and Digestive and Kidney Diseases (R01DK100790) and the National Institute of General Medical Sciences (U54GM104940) of the National Institutes of Health.

\section{References}

1. Cho N, Shaw J, Karuranga S, et al. IDF Diabetes Atlas: Global estimates of diabetes prevalence for 2017 and projections for 2045. 2018.

2. Xu Y, Wang L, He J, et al. Prevalence and control of diabetes in Chinese adults. Jama. 2013;310(9): 948-959. [PubMed: 24002281]

3. Metzger BE, Coustan DR. Summary and recommendations of the Fourth International WorkshopConference on Gestational Diabetes Mellitus. The Organizing Committee. Diabetes care. 1998;21 Suppl 2:B161-167. [PubMed: 9704245]

4. Bellamy L, Casas JP, Hingorani AD, Williams D. Type 2 diabetes mellitus after gestational diabetes: a systematic review and meta-analysis. Lancet. 2009;373(9677):1773-1779. [PubMed: 19465232]

5. Collins GS, Mallett S, Omar O, Yu LM. Developing risk prediction models for type 2 diabetes: a systematic review of methodology and reporting. BMC medicine. 2011;9:103. [PubMed: 21902820] 
6. Schmidt MI, Duncan BB, Bang H, et al. Identifying individuals at high risk for diabetes: The Atherosclerosis Risk in Communities study. Diabetes care. 2005;28(8):2013-2018. [PubMed: 16043747]

7. Aekplakorn W, Bunnag P, Woodward M, et al. A risk score for predicting incident diabetes in the Thai population. Diabetes care. 2006;29(8):1872-1877. [PubMed: 16873795]

8. Wilson PW, Meigs JB, Sullivan L, Fox CS, Nathan DM, D'Agostino RB, Sr., Prediction of incident diabetes mellitus in middle-aged adults: the Framingham Offspring Study. Archives of internal medicine. 2007;167(10):1068-1074. [PubMed: 17533210]

9. Chien K, Cai T, Hsu H, et al. A prediction model for type 2 diabetes risk among Chinese people. Diabetologia. 2009;52(3):443-450. [PubMed: 19057891]

10. Hippisley-Cox J, Coupland C, Robson J, Sheikh A, Brindle P. Predicting risk of type 2 diabetes in England and Wales: prospective derivation and validation of QDScore. BMJ. 2009;338:b880. [PubMed: 19297312]

11. Kahn HS, Cheng YJ, Thompson TJ, Imperatore G, Gregg EW. Two risk-scoring systems for predicting incident diabetes mellitus in U.S. adults age 45 to 64 years. Annals of internal medicine. 2009;150(11):741-751. [PubMed: 19487709]

12. Liu H, Zhang C, Zhang S, et al. Prepregnancy body mass index and weight change on postpartum diabetes risk among gestational diabetes women. Obesity (Silver Spring). 2014;22(6):1560-1567. [PubMed: 24616432]

13. Hu G, Tian H, Zhang F, et al. Tianjin Gestational Diabetes Mellitus Prevention Program: study design, methods, and 1-year interim report on the feasibility of lifestyle intervention program. Diabetes research and clinical practice. 2012;98(3):508-517. [PubMed: 23010556]

14. Li W, Zhang S, Liu H, et al. Different associations of diabetes with beta-cell dysfunction and insulin resistance among obese and nonobese Chinese women with prior gestational diabetes mellitus. Diabetes care. 2014;37(9):2533-2539. [PubMed: 24914241]

15. Wang L, Liu H, Zhang S, et al. Obesity index and the risk of diabetes among Chinese women with prior gestational diabetes. Diabetic medicine : a journal of the British Diabetic Association. 2014;31(11):1368-1377. [PubMed: 24961948]

16. Shen Y, Wang P, Wang L, et al. Gestational diabetes with diabetes and prediabetes risks: a large observational study. Eur J Endocrinol. 2018;179:51-58. [PubMed: 29743168]

17. Zhang F, Dong L, Zhang CP, et al. Increasing prevalence of gestational diabetes mellitus in Chinese women from 1999 to 2008. Diabetic medicine : a journal of the British Diabetic Association. 2011;28(6):652-657. [PubMed: 21569085]

18. Alberti KG, Zimmet PZ. Definition, diagnosis and classification of diabetes mellitus and its complications. Part 1: diagnosis and classification of diabetes mellitus provisional report of a WHO consultation. Diabetic medicine : a journal of the British Diabetic Association. 1998;15(7): 539-553. [PubMed: 9686693]

19. American Diabetes A Diagnosis and classification of diabetes mellitus. Diabetes care. 2005;28 Suppl 1:S37-42. [PubMed: 15618111]

20. D'Agostino RB, Sr., Grundy S, Sullivan LM, Wilson P Validation of the Framingham coronary heart disease prediction scores: results of a multiple ethnic groups investigation. Jama. 2001;286(2):180-187. [PubMed: 11448281]

21. Iasonos A, Schrag D, Raj GV, Panageas KS. How to build and interpret a nomogram for cancer prognosis. Journal of clinical oncology : official journal of the American Society of Clinical Oncology. 2008;26(8):1364-1370. [PubMed: 18323559]

22. Tilgner H, Nikolaou C, Althammer S, et al. Nucleosome positioning as a determinant of exon recognition. Nature structural \& molecular biology. 2009;16(9):996-1001.

23. Grimes DA. The nomogram epidemic: resurgence of a medical relic. Annals of internal medicine. 2008;149(4):273-275. [PubMed: 18711159]

24. Graesslin O, Abdulkarim BS, Coutant C, et al. Nomogram to predict subsequent brain metastasis in patients with metastatic breast cancer. Journal of clinical oncology : official journal of the American Society of Clinical Oncology. 2010;28(12):2032-2037. [PubMed: 20308667] 
25. Wang Y, Li J, Xia Y, et al. Prognostic nomogram for intrahepatic cholangiocarcinoma after partial hepatectomy. Journal of clinical oncology : official journal of the American Society of Clinical Oncology. 2013;31(9):1188-1195. [PubMed: 23358969]

26. Balachandran VP, Gonen M, Smith JJ, DeMatteo RP. Nomograms in oncology: more than meets the eye. The Lancet Oncology. 2015;16(4):e173-180. [PubMed: 25846097]

27. Oza-Frank R, Ali MK, Vaccarino V, Narayan KM. Asian Americans: diabetes prevalence across U.S. and World Health Organization weight classifications. Diabetes care. 2009;32(9):1644-1646. [PubMed: 19509010]

28. Shai I, Jiang R, Manson JE, et al. Ethnicity, obesity, and risk of type 2 diabetes in women: a 20year follow-up study. Diabetes care. 2006;29(7):1585-1590. [PubMed: 16801583]

29. WHO Expert C. Appropriate body-mass index for Asian populations and its implications for policy and intervention strategies. Lancet. 2004;363(9403):157-163. [PubMed: 14726171]

30. Torrens JI, Skurnick J, Davidow AL, et al. Ethnic differences in insulin sensitivity and beta-cell function in premenopausal or early perimenopausal women without diabetes: the Study of Women's Health Across the Nation (SWAN). Diabetes care. 2004;27(2):354-361. [PubMed: 14747213]

31. Weng J, Hu G. Diabetes: Leveraging the Tipping Point of the Diabetes Pandemic. Diabetes. 2017;66:1461-1463. [PubMed: 28533297]

32. Kim C, Newton K, Knopp R. Gestational diabetes and the incidence of type 2 diabetes: a systematic review. Diabetes care. 2002;25:1862-1868. [PubMed: 12351492]

33. International Association of Diabetes and Pregnancy Study Groups Consensus Panel, Metzger B, Gabbe $S$, et al. International association of diabetes and pregnancy study groups recommendations on the diagnosis and classification of hyperglycemia in pregnancy. Diabetes care. 2010;33:676682. [PubMed: 20190296] 


\section{What Is Known on This Subject?}

More than 40 risk prediction models for type 2 diabetes have been developed among different populations until now, but only very small minority were routinely used in clinical practice, due to the heterogeneity between different populations and complex mathematical formulas, lack of simple and visual tools to promote the usage of these prediction risk models. Moreover, most of risk prediction models for type 2 diabetes were conducted among general population, and no tools have been developed to predict postpartum type 2 diabetes among women with gestational diabetes. 


\section{What Does This Study Add?}

The present study developed a simple, fast, and noninvasive tool to identify postpartum type 2 diabetes risk among Chinese women with gestational diabetes diagnosed by WHO 1998 criteria. 


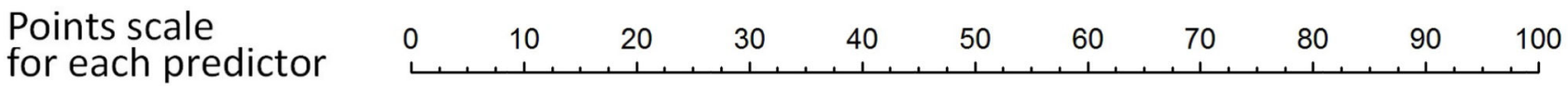

Family history of diabetes

BMI category Before pregnancy

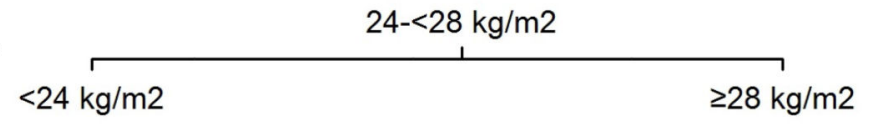

Pregnancy-induced hypertension

2-hour 75-g glucose category at 26-30 gestational weeks

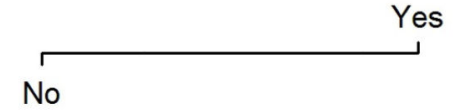

No

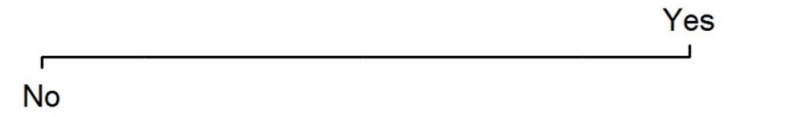

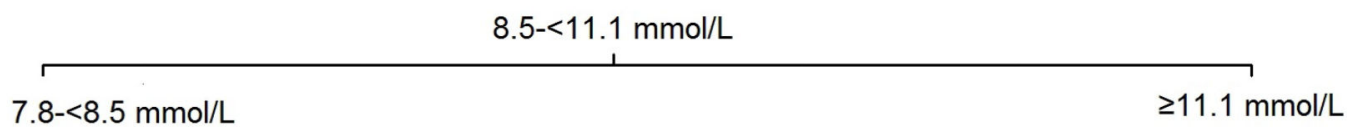

Total Points of predictors

$\begin{array}{llllllllllllll}0 & 20 & 40 & 60 & 80 & 100 & 120 & 140 & 160 & 180 & 200 & 220 & 240 & 260\end{array}$

2-year Incidence Probability scale for total points

3-year Incidence Probability scale for total points
0.01

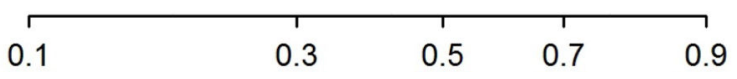

Figure 1.

Nomograms for incident risk of postpartum type 2 diabetes among Chinese women with gestational diabetes mellitus. 

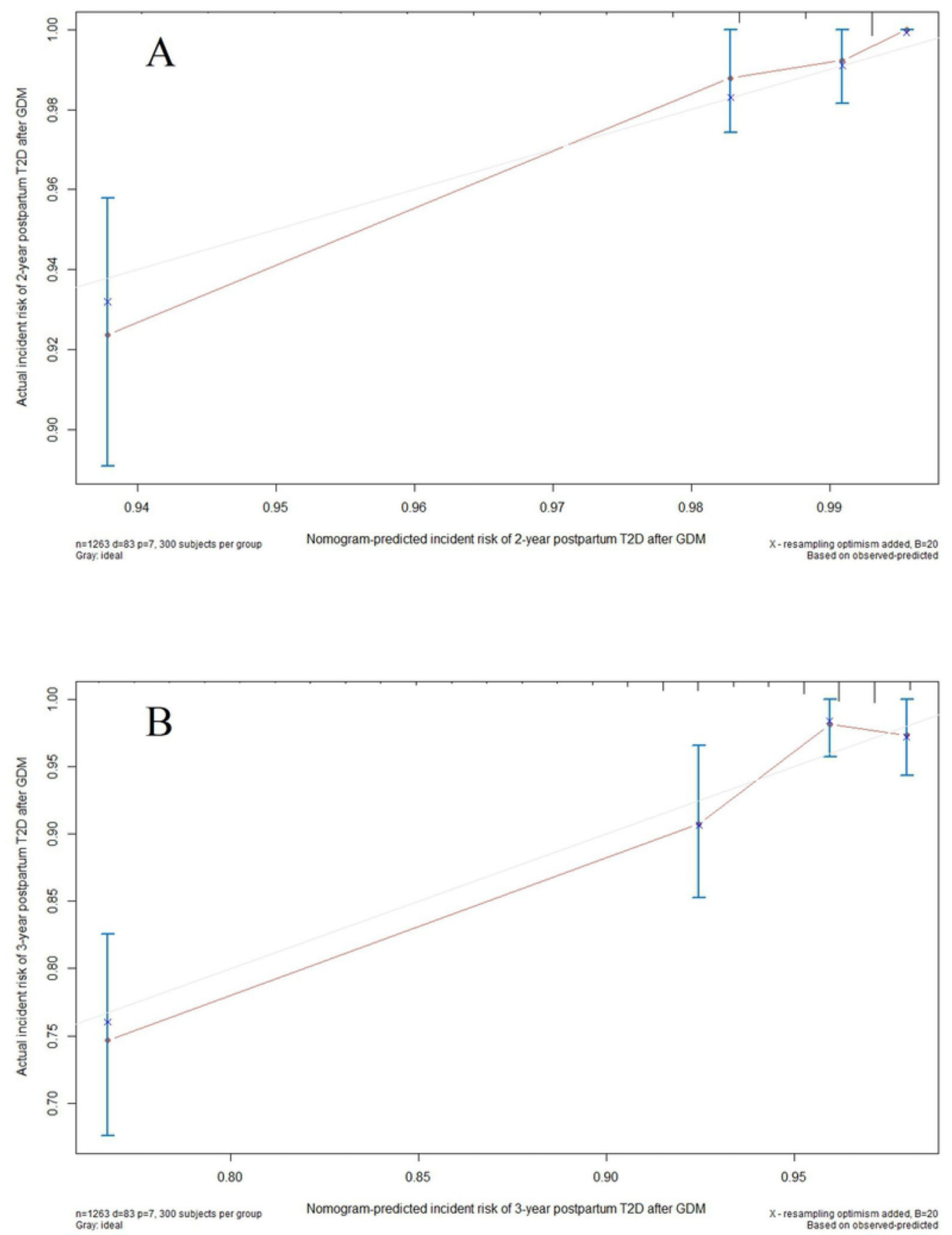

Figure 2.

Calibration plots of nomograms for postpartum 2-year (A) and 3-year (B) risk of type 2 diabetes. 
Table 1.

Characteristics of study samples with and without postpartum diabetes

\begin{tabular}{|c|c|c|c|}
\hline Variables & Non-diabetes $(\mathrm{N}=\mathbf{1 1 8 0})$ & Diabetes $(\mathrm{N}=\mathbf{8 3})$ & P value \\
\hline Postpartum time after delivery, year, M (SE) & $2.26(0.9)$ & $2.63(0.9)$ & $<0.001$ \\
\hline \multicolumn{4}{|l|}{ Variables before/during pregnancy } \\
\hline Family history of diabetes, $\mathrm{N}(\%)$ & $402(34.1)$ & $49(59.0)$ & $<0.001$ \\
\hline Pre-pregnancy body mass index, $\mathrm{kg} / \mathrm{m}^{2}, \mathrm{~N}(\%)$ & & & $<0.001$ \\
\hline$<24$ & $793(67.2)$ & $30(36.1)$ & \\
\hline 24-27.9 & $303(25.7)$ & $32(38.6)$ & \\
\hline$\geq 28$ & $84(7.1)$ & $21(25.3)$ & \\
\hline Age at pregnancy, year, $\mathrm{M}(\mathrm{SD})$ & $30.1(3.5)$ & $30.0(3.8)$ & 0.857 \\
\hline Pregnancy-induced hypertension, $\mathrm{N}(\%)$ & $70(5.9)$ & $21(25.3)$ & $<0.001$ \\
\hline 2-hour 75-g glucose level at 26-30 gestational weeks, mmol/L, $\mathrm{N}(\%)$ & & & $<0.001$ \\
\hline $7.8-8.5$ & 455 (38.6) & $10(12.1)$ & \\
\hline $8.5-11.1$ & $647(54.8)$ & 45 (54.2) & \\
\hline$\geq 11.1$ & $78(6.6)$ & 28 (33.7) & \\
\hline Weight gain during pregnancy ${ }^{*}, \mathrm{~N}(\%)$ & & & 0.126 \\
\hline Inadequate & $161(13.6)$ & 18 (21.7) & \\
\hline Adequate & $366(31.0)$ & 24 (28.9) & \\
\hline Excessive & $653(55.3)$ & $41(49.4)$ & \\
\hline
\end{tabular}

* IOM categories: Inadequate: $<12.5 \mathrm{~kg}$ (pre-pregnancy BMI $<18.5 \mathrm{~kg} / \mathrm{m}^{2}$ ), $<11.5 \mathrm{~kg}$ (BMI $18.5-23.9 \mathrm{~kg} / \mathrm{m}^{2}$ ), $<7 \mathrm{~kg}$ (BMI $24.0-27.9 \mathrm{~kg} / \mathrm{m}^{2}$ ), and $<5 \mathrm{~kg}\left(\mathrm{BMI}>28 \mathrm{~kg} / \mathrm{m}^{2}\right)$; Adequate: $12.5-18 \mathrm{~kg}\left(\right.$ BMI $\left.<18.5 \mathrm{~kg} / \mathrm{m}^{2}\right), 11.5-16 \mathrm{~kg}$ (BMI 18.5- 23.9 kg/m²), 7-11.5 kg (BMI 24.0-27.9 kg/m $\left.{ }^{2}\right)$, and

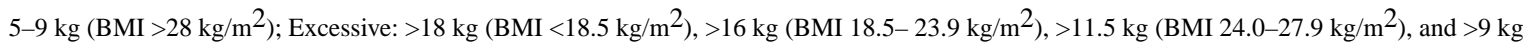
$\left(\right.$ BMI $>28 \mathrm{~kg} / \mathrm{m}^{2}$ ), according to the Chinese maternal pre-pregnancy BMI classification standard and the 2009 IOM GWG recommendations. 


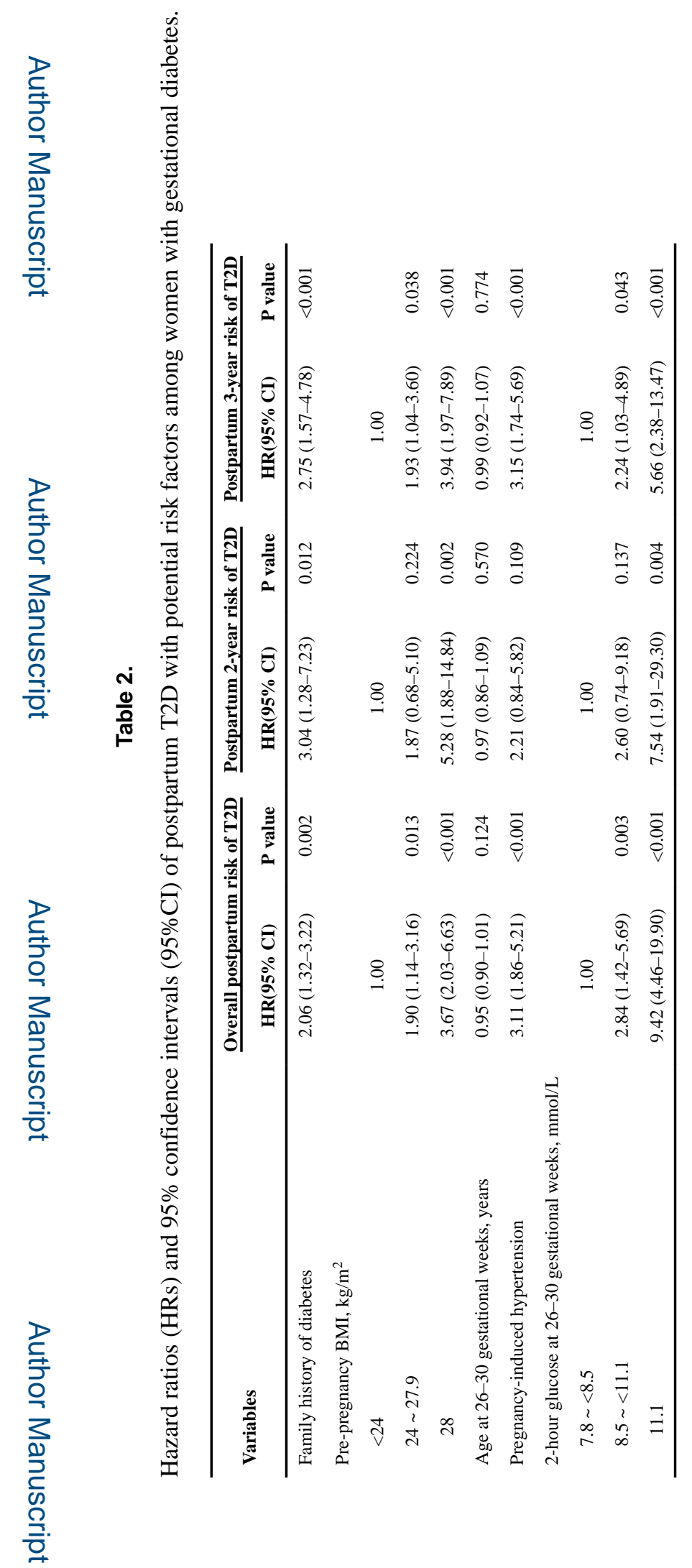

Clin Endocrinol (Oxf). Author manuscript; available in PMC 2020 March 01. 